\title{
Characterization of the melanopsin gene (Opn4x) of diurnal and nocturnal snakes
}

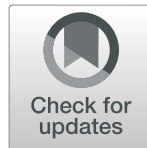

Einat Hauzman ${ }^{1,2^{*}}$ (D, Venkatasushma Kalava ${ }^{3}$, Daniela Maria Oliveira Bonci ${ }^{1,2}$ and Dora Fix Ventura ${ }^{1,2}$

\begin{abstract}
Background: A number of non-visual responses to light in vertebrates, such as circadian rhythm control and pupillary light reflex, are mediated by melanopsins, G-protein coupled membrane receptors, conjugated to a retinal chromophore. In non-mammalian vertebrates, melanopsin expression is variable within the retina and extra-ocular tissues. Two paralog melanopsin genes were classified in vertebrates, Opn4x and Opn4m. Snakes are highly diversified vertebrates with a wide range of daily activity patterns, which raises questions about differences in structure, function and expression pattern of their melanopsin genes. In this study, we analyzed the melanopsin genes expressed in the retinas of 18 snake species from three families (Viperidae, Elapidae, and Colubridae), and also investigated extra-retinal tissue expression.

Results: Phylogenetic analysis revealed that the amplified gene belongs to the Opn4x group, and no expression of the Opn4m was found. The same paralog is expressed in the iris, but no extra-ocular expression was detected. Molecular evolutionary analysis indicated that melanopsins are evolving primarily under strong purifying selection, although lower evolutionary constraint was detected in snake lineages $(\omega=0.2)$, compared to non-snake Opn $4 x$ and Opn4m $(\omega=0.1)$. Statistical analysis of selective constraint suggests that snake phylogenetic relationships have driven stronger effects on melanopsin evolution, than the species activity pattern. In situ hybridization revealed the presence of melanopsin within cells in the outer and inner nuclear layers, in the ganglion cell layer, and intense labeling in the optic nerve.

Conclusions: The loss of the Opn4m gene and extra-ocular photosensitive tissues in snakes may be associated with a prolonged nocturnal/mesopic bottleneck in the early history of snake evolution. The presence of melanopsincontaining cells in all retinal nuclear layers indicates a globally photosensitive retina, and the expression in classic photoreceptor cells suggest a regionalized co-expression of melanopsin and visual opsins.
\end{abstract}

Keywords: Melanopsin, Photopigment, Snakes, Retina, Circadian rhythm, dN/dS

\section{Background}

For their survival, animals depend on the ability to capture and process information from the surrounding environment. Information acquired through light processing channels is captured by photoreceptor cells, whose photopigments absorb photons and lead to the generation of neural responses to light. Responses to light by photoreceptor cells may trigger circuits dedicated to visual processing and image formation or may activate neural

\footnotetext{
* Correspondence: hauzman.einat@gmail.com

'Departamento de Psicologia Experimental, Instituto de Psicologia,

Universidade de São Paulo, Av. Professor Mello Moraes, 1721, Bloco A - Sala

D9. Butantã, São Paulo, SP 05508-030, Brazil

${ }^{2}$ Instituto Israelita de Ensino e Pesquisa Albert Einstein, São Paulo, Brazil

Full list of author information is available at the end of the article
}

circuits with non-visual functions, such as circadian rhythm control, pupil light reflex, and skin color changes [1]. The visual process is initiated by visual photopigments, opsins and rhodopsins, coupled to a retinal chromophore, located in rods and cones. Non-visual responses to light rely on the activity of a number of opsin classes, for instance, encephalopsins, parapinopsins, and melanopsins [2-6]. Non-visual opsins may be located in the retina and iris, and in extra-ocular photosensitive tissues, such as skin, pineal gland, brain, and liver, depending on the opsin group and vertebrate class [7].

Opsins are membrane receptors that belong to the G-protein-coupled receptor family, with seven transmembrane domains, characterized by their ability to bind a vitamin Abased chromophore, usually the 11-cis-retinal, via a Schiff 
base linkage in a conserved Lysine residue, in the seventh transmembrane $\alpha$-helix. Absorption of a photon by the chromophore causes its photoisomerization from 11-cis to all-trans form, which leads to conformational changes of the opsin, allowing a G-protein to bind and activate the phototransduction cascade within the photoreceptor cell [8-10]. This process leads to the closure or opening of ion channels in the plasma membrane, depending on the photoreceptor cell type, and the photoreceptor's electrical response to light $[11,12]$.

In the 1990s, the discovery of a new photopigment expressed in dermal melanophores of the frog Xenopus laevis [5] boosted new investigations for the understanding of the functions and the possible relationship of these photopigments with non-visual responses to light. This newly discovered photopigment, named melanopsin, was then identified in the inner retinas of mammals [6]. Studies with knockout mice models revealed the role of this photopigment in the circadian rhythm control [13-16] and following studies showed that melanopsins are responsible for other non-visual responses to light, such as pupillary light reflex, melatonin suppression, and skin color changes [17-27].

Comparative studies on the melanopsin gene have shown the presence of two paralogs within vertebrate genomes, the Xenopus-like $(\mathrm{Opn} 4 \mathrm{x})$ and the mammalian-like $(\mathrm{Opn} 4 \mathrm{~m})$ [28], which were originated from a duplication event in the early history of vertebrates evolution [29]. In mammals, only the $\mathrm{Opn} 4 m$ gene is expressed in a subset of intrinsically photosensitive retinal ganglion cells (ipRGCs) [30]. Nonmammalian vertebrates may express both $\mathrm{Opn} 4 m$ and $O p n 4 x$ genes, with varied tissue expression patterns $[5,28$, 31-38]. The loss of the Opn4x paralog in the mammalian genome and the loss of extra-ocular photosensitive tissues was associated with a "nocturnal bottleneck" in the early history of mammalian evolution [39]. On the other hand, in teleost fish, later duplication events led to new melanopsin paralogs expressed in different classes of retinal neurons, and with distinct functional properties [40, 41].

The role of the Opn4m photopigment in mediating photoentrainment, circadian rhythm regulation, melatonin suppression and pupillary light reflex, has been demonstrated in mammals $[14,16-18,20,23$, 42, 43]. However, the specific functions of each melanopsin paralog in non-mammalian vertebrates still need extensive investigation in the context of its complex expression pattern. For instance, in chicken, variations of $O p n 4 x$ and $O p n 4 m$ expression levels and location within the retina were observed during different stages of development and throughout the day [44]. Whereas Opn4m expression is restricted to a subpopulation of ganglion cells during all life stages, Opn $4 x$ expression is limited to ipRGCs and the optic nerve only at early embryonic stages, and later it is expressed in horizontal cells, with daily rhythmic expression levels in the mature retina $[34,35,44,45]$.

In reptiles, melanopsin expression has been characterized in a few species, including the freshwater red ear turtle Trachemys scripta elegans, which expresses the Opn4m in the retina [46], the ruin lizard Podarcis sicula, which expresses the $O p n 4 x$ in the retina, lateral eye and brain [47], and in sea snakes, which express the $O p n 4 x$ in the skin [48]. In the Elapidae sea-snakes Aipysurus laevis and A. tenuis, the expression of melanopsin in the skin was associated to dermal phototaxis of the tail, a behavior observed in this group of marine snakes [48]. Genome data [49-51] and eye transcriptome analysis [52], have indicated that snakes have lost the Opn4m gene prior to the origin of Serpentes [50] and had only kept the Opn4x paralog.

The suborder Serpentes comprises a highly diversified group of vertebrates and represents a valuable model to investigate specific functions and expression patterns of photosensitive proteins, which may contribute to the understanding of the evolution of vertebrate circadian timing regulation. The diversity of the group, with more than 3700 living species [53] exhibits a number of adaptations to their ecological niches, which include distinct daily activity patterns [54-57]. These adaptations raise intriguing questions about selective pressure acting on specific genes related to circadian activity control. In this study, we isolated the melanopsin gene of diurnal and nocturnal snakes from different families, determined its phylogenetic position, and characterized its tissue-specific expression pattern, and retinal location. We also investigated selection patterns of molecular evolution in a phylogenetic framework, using several models to detect selection signals in the protein level. Our results bring new light and increase the knowledge on the evolution of melanopsins and photosensory systems in reptiles.

\section{Results \\ Gene identification and phylogenetic position of melanopsin of snakes}

Partial sequences of the melanopsin gene ( $1400 \mathrm{bp})$ of 18 snake species (Additional file 1: Table S1) were successfully amplified with the primers designed based on snake melanopsin sequences. No amplification was observed in PCRs performed with primers designed based on the Opn $4 m$. All sequences have been deposited at GenBank (accession numbers: MN241125- MN241142) (Additional file 1: Table S1).

The amplified fragments possessed the general features of melanopsins and the G-protein-coupled receptor proteins. Alignment of the amino acid sequences reveals some conserved key features, including a Lysine residue $(284 \mathrm{~K})$, located in the seventh transmembrane domain, 
that acts as the retinal attachment site through a Schiff base [9], and a Tyrosine located in the equivalent position of the Glutamate Schiff base counterion of visual opsins (Y92) (Additional file 2). Other conserved features include a Glutamate (E) at residue 161, which serve as possible counterion site [58, 59], two Cysteine (C) residues at sites 89 and 167 for disulfide bond formation, and the D113/ R114/Y115 motif in the third transmembrane for G protein binding [60] (Additional file 2).

The final alignment used for phylogenetic reconstruction was 2397 base pairs long. ML reconstruction recovered the monophyly of the two melanopsin genes, Opn4m and Opn4x (bootstrap supports: 99 and 78, respectively), and grouped the melanopsins of snakes within the Opn $4 x$ group, with high bootstrap support (89) (Fig. 1). The melanopsin tree was highly consistent with snake phylogenies [61] and recovered the monophyly of the three caenophidian families sampled, Viperidae, Elapidae, and Colubridae. In most cases, the topology of internal branches were highly consistent with species phylogeny, with only exception for internal branches of the Colubrinae subfamily [61] (Fig. 1).

\section{Statistical analysis of molecular evolution}

We investigated the patterns of selection in snake melanopsins using codon-based likelihood models to estimate $\mathrm{d}_{\mathrm{N}} / \mathrm{d}_{\mathrm{S}}$. For branch model analysis, we used a broad data set with 107 sequences including the Opn4m and Opn4x genes from different vertebrates, to estimate the overall selective pattern of melanopsins (Additional file 1: Table S2; Sequences alignment: Additional file 3). For random site, branch-site and clade models, we used a smaller data set containing 29 snake melanopsin coding sequences, 18 of which generated in this study (Fig. 2; Sequences alignment: Additional file 4).

Statistical analysis of branch models showed an overall low $\omega$ rate under the null model $(\omega=0.107)$, which indicates a strong evolutionary constraint on the melanopsin genes (Table 1). Likelihood ratio tests (LRTs) showed that the two-partition model, with independent $\omega$ values for Opn4m $(\omega=0.097)$ and Opn4x $(\omega=0.125)$, and the three-partition model, with independent $\omega$ values for Opn4m, non-snakes Opn4x $(\omega=0.114)$, and snakes Opn $4 x(\omega=0.204)$, were significantly better than the null $(1 \omega)$ model (Table 1$)$.

The LRT comparison of random-site models showed significant difference between M0 and M3 and between M7/M8 and M8a/M8 models (Table 2), indicating that relative rates of substitution are variable among sites. Models M2a, M3 and M8 had a significantly better fit than the alternative models $(p<0.05)$, and several sites were indicated by BEB as under positive selection (Additional file 1: Table S3).

We used clade models to investigate the effects of species phylogeny and daily activity pattern on the evolution of snake melanopsins. Different $\mathrm{CmC}$ partition models were implemented to test whether diurnal or nocturnal activities may have driven divergences in melanopsin evolution. LRTs showed that all implemented partitions considering both phylogeny and daily activity pattern were better than the null model, M2a_rel (Table 3). Two different partition models were tested to investigate the effects of daily activity pattern, a two-partition which isolates diurnal snakes from all nocturnal lineages, and a three-partition which separated the henophidian snake Python bivitattus in one clade, and diurnal and nocturnal caenophidian species in independent foreground clades (Fig. 2). LRT comparisons indicated that the three-partition model, was significantly better than the two-partition model, and diurnal lineages had lower $\mathrm{d}_{\mathrm{N}} /$ $\mathrm{d}_{\mathrm{S}}$ value $(\omega=0.06)$ compared to nocturnal $(\omega=0.2)$ (Table 3). According to LRT and BIC comparisons, the best fitting model overall was the phylogenetic four-partition model which isolates each snake family in a separate foreground branch, with lower $\omega$ value for Colubridae snakes $(\omega=0.03)$ (Table 3$)$. The results from $\mathrm{CmC}$ models suggest that although daily activity pattern seems to apply different constrains on the evolution of melanopsin gene in snakes, with stronger signals of purifying selection in diurnal lineages, snakes phylogenetic relationships have driven stronger effects on Opn $4 x$ evolution.

To investigate for positive selective sites in specific clades, we used branch-site models, isolating independently diurnal and nocturnal caenophidian lineages in foreground clades, and the three families within the Caenophidia group, Viperidae, Elapidae, and Colubridae. Based on the results from $\mathrm{CmC}$ models, we also performed branch-site models isolating diurnal and nocturnal snakes from the Colubridae family, to investigate specific divergent selection in this particular group. The LRT and BIC comparisons indicated that the model designating primarily diurnal snake lineages as foreground branch was slightly better than the null model $(p=$ 0.048; Additional file 1: Table S4), and BEB analysis indicated only two sites under positive selection $\left(\omega_{2}=3.3\right.$, $\mathrm{p}_{2}+\mathrm{p}_{3}=0.02$; Additional file 1: Table S4). In addition, branch-site model considering the Colubridae family as foreground branch was better than the null model $(p<$ 0.05; $\omega_{2}=3.7, \mathrm{p}_{2}+\mathrm{p}_{3}=0.05$; Additional file 1: Table S4), such as the model designating diurnal Colubridae snakes as foreground branch $\left(p=0.003 ; \omega_{2}=4.2, \mathrm{p}_{2}+\mathrm{p}_{3}=0.03\right.$; Additional file 1: Table S4).

\section{Expression pattern and melanopsins location within the retinas}

Analysis of extra-retinal melanopsin expression revealed the expression of the same melanopsin gene in the iris of three colubrid species, T. dorsatus, S. neuwiedi, and 


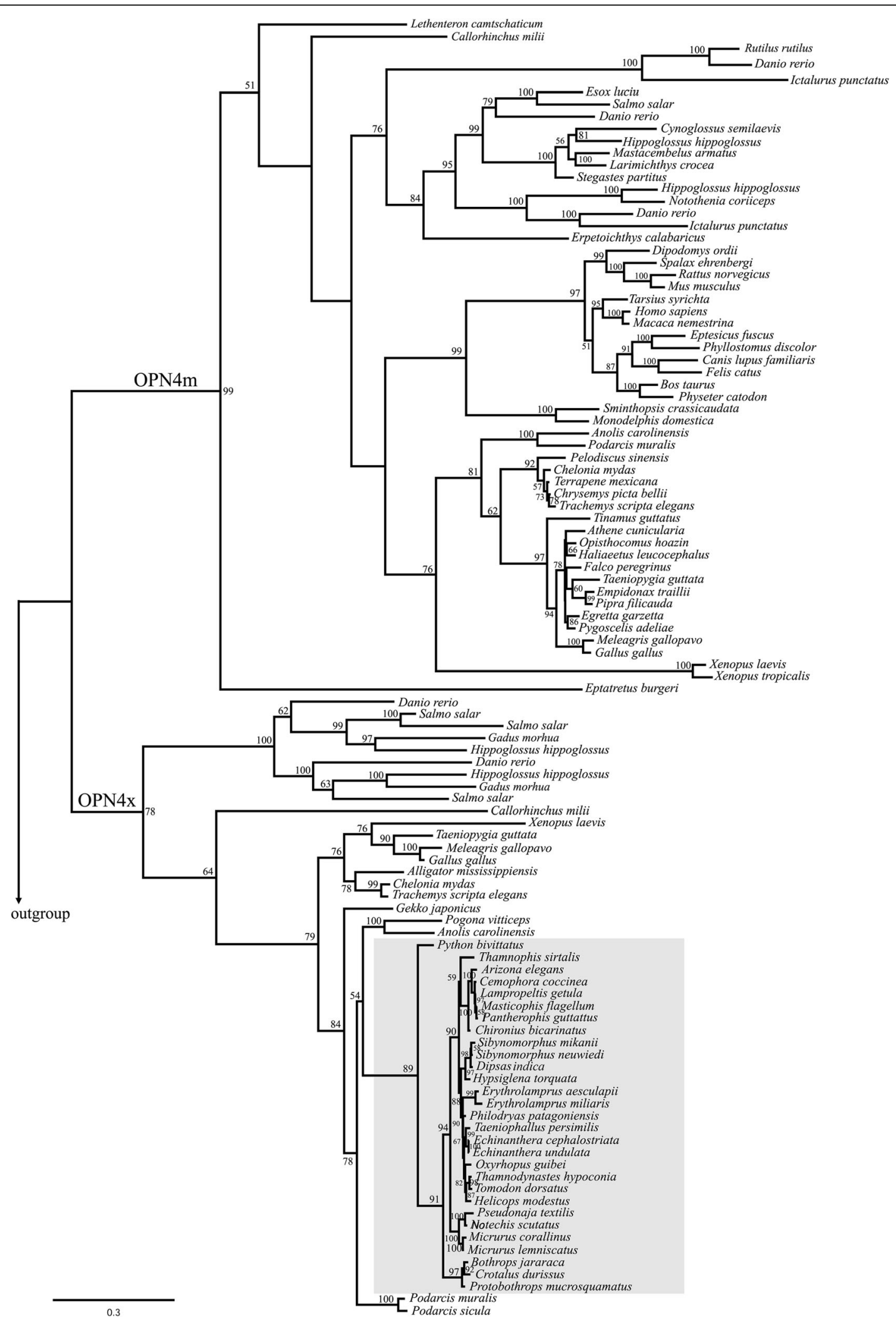

Fig. 1 Phylogenetic reconstruction of vertebrate melanopsin genes Opn4m and Opn4x, by Maximum Likelihood (ML). Snake melanopsin sequences (gray box) correspond to the Opn4x gene. The amphioxus melanopsin sequence (GenBank: Q4R1/4) was used as outgroup (not shown). ML bootstrap supports are indicated for each resolved node. The scale bar represents the number of nucleotide substitutions per site 


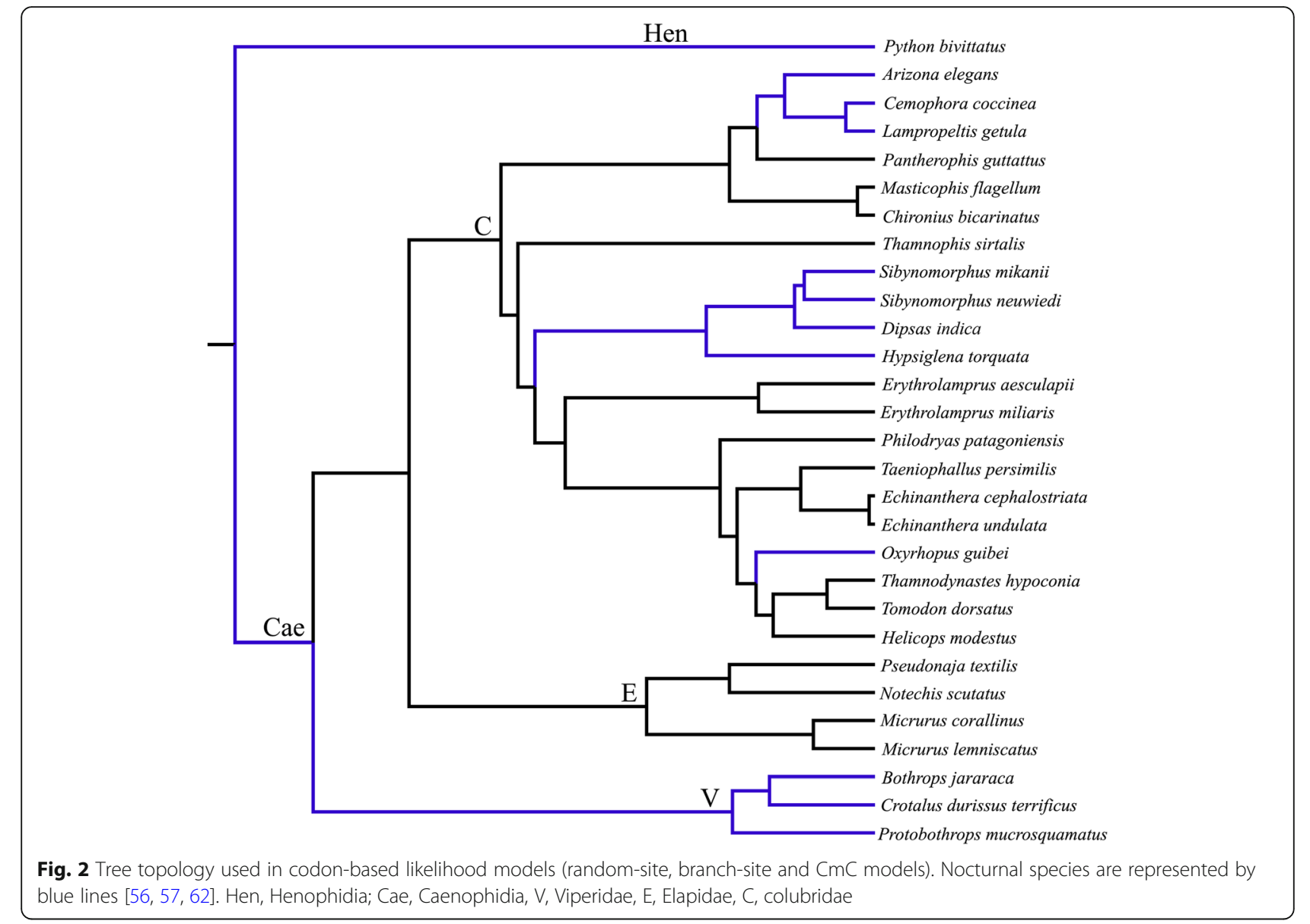

O. guibei. No expression was detected in extra-ocular tissues. In situ hybridization revealed melanopsin expression in the retinal outer nuclear layer, i.e., photoreceptors nuclei, in the inner nuclear layer, in contact with the inner plexiform layer, and in the ganglion cell layer, indicating the presence of ipRGCs (Fig. 3a). Labeling was observed in the retinal edge, but not in retinal core regions. Intense labeling was observed in the optic nerve (Fig. 3b).

\section{Discussion}

This study provides new evolutionary information on the melanopsin gene family and specifically characterizes the Opn $4 x$ gene and its expression pattern in the retinas of snakes from three different families. The highly diverse class of reptiles is still misrepresented in studies on visual and non-visual photopigments; our analysis provides new information on the characteristics and variability of photoreceptor genes in this group.

The phylogenetic analysis showed that the melanopsin gene of snakes belongs to the Opn $4 x$ group. The tree topology was highly consistent with snake phylogeny [61]. The branch of snakes formed a monophyletic clade with those of other squamate reptiles. The molecular evolutionary analysis indicates that the melanopsin gene is under evolutionary constraint in snake lineages. Borges et al. [29] found that amino acid substitutions in melanopsins of vertebrates are mainly under negative selection $(\omega=0.17)$, which indicates the important physiological role of this protein for the organism fitness [29]. However,

Table 1 Parameter estimates and log likelihood values under branch models for the melanopsin genes Opn4m and Opn4x

\begin{tabular}{|c|c|c|c|c|c|c|c|c|c|}
\hline Branch Model & $\mathrm{np}$ & $\ln \mathrm{L}$ & K & Parameters & $\mathrm{BIC}$ & & LRT & $\mathrm{df}$ & $P$ \\
\hline $1 \omega$ & 213 & $-28,548.3$ & 2.0 & $\omega=0.107$ & $57,624.8$ & - & & & \\
\hline $2 \omega$ & 214 & $-28,539.9$ & 2.0 & OPN4m: $\omega=0.097 ;$ OPN4x: $\omega=0.125$ & $57,601.5$ & $1 \omega$ & 17 & 1 & 0.000 \\
\hline \multirow[t]{2}{*}{$3 \omega$} & 215 & $-28,529.4$ & 2.0 & OPN4m: $\omega=0.097 ;$ OPN4x: $\omega=0.11 ;$ Snakes-OPN4x: $\omega=0.20$ & $57,591.9$ & $2 \omega$ & 21 & 1 & 0.000 \\
\hline & & & & & & $1 \omega$ & 38 & 2 & 0.000 \\
\hline
\end{tabular}


Table 2 Likelihood ratio tests for Random-Sites Models of Snake Opn4x Species Tree

\begin{tabular}{|c|c|c|c|c|c|c|c|c|}
\hline Model & $\mathrm{np}$ & $\ln L$ & K & Parameters & & LRT & df & p \\
\hline M0 & 57 & -6516.8 & 3.4 & $\omega_{0}=0.40$ & & & & \\
\hline M1a & 58 & -6417.8 & 3.4 & $\omega_{0}=0.08\left(p_{0}=0.68\right) ; \omega_{1}=1.0(0.32)$ & & & & \\
\hline $\mathrm{M} 2 \mathrm{a}$ & 60 & -6411.7 & 3.5 & $\omega_{0}=0.1\left(p_{0}=0.69\right) ; \omega_{1}=1.0\left(p_{1}=0.26\right) ; \omega_{2}=2.45\left(p_{2}=0.05\right)$ & M1a & 12.2 & 2 & 0.002 \\
\hline M3 & 61 & -6409.6 & 3.5 & $\omega_{0}=0.0\left(p_{0}=0.4\right) ; \omega_{1}=0.43\left(p_{1}=0.48\right) ; \omega_{2}=1.93\left(p_{2}=0.12\right)$ & M0 & 214.4 & 4 & 0.000 \\
\hline M7 & 58 & -6421.1 & 3.4 & $p=0.17 ; q=0.28$ & & & & \\
\hline M8a & 59 & -6417.7 & 3.4 & $\mathrm{p}_{0}=0.69, p=1.57, \mathrm{q}=14.77\left(\mathrm{p}_{1}=0.31, \omega=1.0\right)$ & & & & \\
\hline \multirow[t]{2}{*}{ M8 } & 60 & -6410.1 & 3.5 & $p_{0}=0.91, p=0.36, q=0.96\left(p_{1}=0.09, \omega=2.09\right)$ & M7 & 22.1 & 2 & 0.000 \\
\hline & & & & & M8a & 15.2 & 1 & 0.000 \\
\hline
\end{tabular}

$n p$ number of parameters, In $L$ In likelihood, $\kappa$ transition/transversion ratio, $L R T$ likelihood ratio test, $d f$ degrees of freedom

in our analysis, we found evidence for lower functional constraint in snakes, with higher $\mathrm{d}_{\mathrm{N}} / \mathrm{d}_{\mathrm{S}}$ ratio $(\omega=0.204)$, compared to non-snakes Opn $4 x(\omega=0.114)$ and $O p n 4 m$ $(\omega=0.097)$ (Table 1). This more relaxed evolutionary pressure in snake $O p n 4 x$ is intriguing. The melanopsin paralog Opn4m seems to be absent in the snake genome [49-51], and one would expect that the presence of only one melanopsin copy would imply stronger constraints in the evolution of the remaining gene, to preserve the structure and function of this protein, necessary for a number of physiological functions driven by responses to light.

Previous studies investigated the expression of visual opsin genes in snakes [62-67]. Three classes of visual opsin genes are expressed in retinas of most snakes studied so far: the rhodopsin gene $R h 1$, the short wavelength sensitive opsin gene Sws1, and the long wavelength sensitive opsin gene $L w s$ [63]. The $R h 2$ and Sws2 genes, sensitive to middle and short wavelengths, are not expressed in snake retinas, and it was suggested that they might have been lost in the ancestor of extant snakes $[49,50,62-64]$. This loss was attributed to nocturnal conditions of ancestral lineages [50-52, 63, 64], as suggested for mammals, which passed through a noctur$\mathrm{nal} /$ mesopic bottleneck [39]. In crocodilians, the loss of two cone opsin genes, Sws 1 and $R h 2$, and inactivating mutations found in Opn4m, were also associated with long periods of adaptation to dim-light conditions in ancestral lineages [68].

Table 3 Clade Model Tests $(\mathrm{CmC})$ for divergence partitioned by snakes phylogeny and by daily activity pattern

\begin{tabular}{|c|c|c|c|c|c|c|c|c|c|}
\hline Model & $\mathrm{np}$ & $\ln L$ & K & Parameters & $\mathrm{BIC}$ & & LRT & $\mathrm{df}$ & $P$ \\
\hline M2_rel & 60 & -6417.6 & 3.38 & $\begin{array}{l}\omega_{0}=0.00\left(p_{0}=0.24\right) ; \\
\omega_{1}=1.0\left(p_{1}=0.30\right) ; \\
\omega_{2}=0.15\left(p_{2}=0.46\right)\end{array}$ & $12,996.9$ & - & & & \\
\hline \multirow[t]{4}{*}{ CmC: 4 part (phylo) } & \multirow[t]{4}{*}{63} & \multirow[t]{4}{*}{-6399.2} & \multirow[t]{4}{*}{3.36} & \multirow{4}{*}{$\begin{array}{l}\omega_{0}=0.00\left(p_{0}=0.26\right) ; \\
\omega_{1}=1.0\left(p_{1}=0.31\right) ; \\
\omega_{2}=0.19\left(p_{2}=0.45\right) ; \text { Hen: } \\
\omega=0.58 ; \text { Vip: } \omega=0.34 ; \text { Elap: } \\
\omega=0.19 ; \text { Col: } \omega=0.03(p=0.44)\end{array}$} & \multirow[t]{4}{*}{$12,968.2$} & M2_rel & 36.8 & 3 & 0.000 \\
\hline & & & & & & 3 part (phylo) & 5.36 & 1 & 0.021 \\
\hline & & & & & & 3 part $(\mathrm{D} \times \mathrm{N})$ & 11.7 & 1 & 0.001 \\
\hline & & & & & & 2 part (phylo) & 16.9 & 2 & 0.000 \\
\hline \multirow[t]{3}{*}{ CmC: 3 part (phylo) } & \multirow[t]{3}{*}{62} & \multirow{3}{*}{-6401.9} & \multirow[t]{3}{*}{3.38} & \multirow{3}{*}{$\begin{array}{l}\omega_{0}=0.03\left(p_{0}=0.4\right) ; \\
\omega_{1}=1.0\left(p_{1}=0.3\right) ; \text { Hen: } \\
\omega=0.84 ; \text { Vip: } \\
\omega=0.5 ; \text { Col+Elap: } \\
\omega=0.03(p=0.3)\end{array}$} & \multirow[t]{3}{*}{$12,970.9$} & M2_rel & 31.5 & 2 & 0.000 \\
\hline & & & & & & 2 part (phylo) & 11.5 & 1 & 0.001 \\
\hline & & & & & & 2 part $(\mathrm{D} \times \mathrm{N})$ & 14.3 & 1 & 0.000 \\
\hline CmC: 2 part (phylo) & 61 & -6407.6 & 1.75 & $\begin{array}{l}\omega_{0}=0.00\left(p_{0}=0.29\right) ; \\
\omega_{1}=1.0\left(p_{1}=0.30\right) ; \text { Hen: } \\
\omega=0.63 ; \text { Caen: } \\
\omega=0.11(p=0.46)\end{array}$ & $12,979.7$ & M2_rel & 19.9 & 1 & 0.000 \\
\hline \multirow[t]{3}{*}{$\mathrm{CmC} 3$ part $(\mathrm{D} \times \mathrm{N})$} & \multirow[t]{3}{*}{62} & \multirow[t]{3}{*}{-6404.8} & \multirow[t]{3}{*}{3.37} & \multirow{3}{*}{$\begin{array}{l}\omega_{0}=0.00\left(p_{0}=0.26\right) ; \\
\omega_{1}=1.0\left(p_{1}=0.29\right) ; \text { Hen: } \\
\omega_{2}=0.56 ; N: \omega=0.2 ; D: \\
\omega=0.06(p=0.45)\end{array}$} & \multirow[t]{3}{*}{$12,977.3$} & M2_rel & 25.1 & 2 & 0.000 \\
\hline & & & & & & 2 part (phylo) & 5.7 & 1 & 0.017 \\
\hline & & & & & & 2 part $(\mathrm{D} \times \mathrm{N})$ & 7.7 & 1 & 0.004 \\
\hline $\mathrm{CmC} 2$ part $(\mathrm{D} \times \mathrm{N})$ & 61 & -6408.9 & 3.38 & $\begin{array}{l}\omega_{0}=0.00\left(p_{0}=0.19\right) ; \\
\omega_{1}=1.0\left(p_{1}=0.3\right) ; N: \\
\omega_{2}=0.24 ; D: \\
\omega=0.05(p=0.51)\end{array}$ & $12,982.2$ & M2_rel & 17.4 & 1 & 0.000 \\
\hline
\end{tabular}




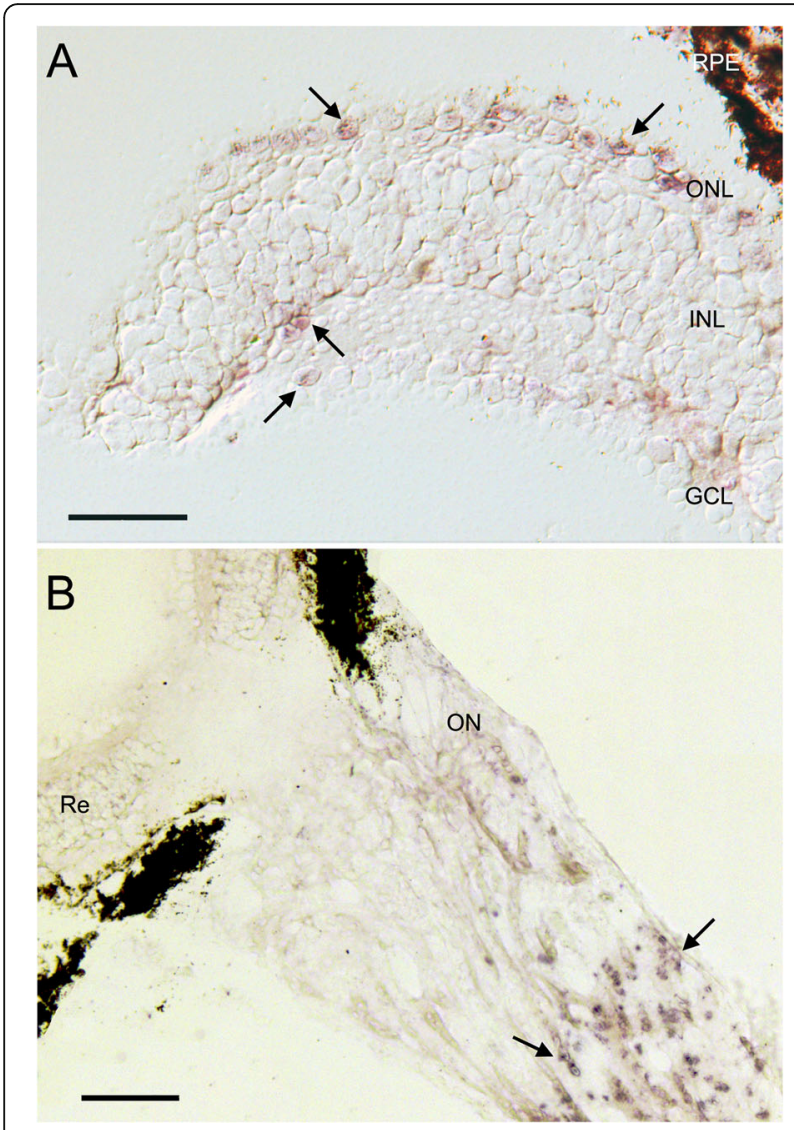

Fig. 3 In situ hybridization using 'antisense' 'DIG' labeled CRNA probes in retinal sections of the diurnal colubrid Tomodon dorsatus. a Expression of melanopsin mRNA in the outer nuclear layer $(\mathrm{ONL})$, in the inner nuclear layer (INL), and ganglion cell layer (GCL) (arrows). b Intense melanopsin expression in the optic nerve (ON) (arrows). No labeling was observed in the retinal sections incubated with 'sense' probes (not shown). RPE, retinal pigment epithelium; Re, retina. Scale bars: $A=50 \mu \mathrm{m}, B=100 \mu \mathrm{m}$

Snakes also seem to have lost other non-visual opsin genes [49-52], and extra-ocular photosensitive tissues, as observed in our analysis, which we may also associate to nocturnal conditions of ancestral lineages [50, 52]. A nocturnal/mesopic bottleneck in the early history of snake evolution may explain the absence of extra-ocular melanopsin expression, analogous to what has been suggested for mammals [29, 69]. However, in the Elapidae sea snakes Aipysurus laevis and $A$. tenuis, two non-visual photopigments were identified from skin transcriptome analysis, the melanopsin gene $O p n 4 x$ and the neuropsin gene Opn5 [48]. The expression of these opsins may be associated with dermal phototaxis in this group of snakes [48], a behavior commonly observed in elongated aquatic vertebrates [70-72]. In our analysis no expression of melanopsin genes were detected in the skin or other extra-ocular tissues.

Our statistical analysis of molecular evolution indicated that species relationships have driven stronger evolutionary constraint on the evolution of melanopsins in snakes, than the species circadian rhythm. Previous studies identified relevant evolutionary features on the visual opsins of snakes, related to the species circadian activity pattern, and different evolutionary constraints were verified among diurnal and nocturnal species [62, 65-67]. Diurnal colubrids have pure cone retinas, with no typical rod-like photoreceptor [39, 73-77], but express the rhodopsin RH1 photopigment, usually expressed in rods [62, 65-67]. Some studies showed evidence that a group of small single cones of diurnal colubrids expresses the RH1 photopigment [65-67], with a considerable blue shift in the absorption peak. Those adaptations reveal the relevance of the circadian activity in the structure, function, and evolution of photopigments of snakes.

Clade models and branch-site models indicated that diurnal activity may have influenced the magnitude of selection acting on the melanopsin gene of snakes, and random site model pointed a number of sites under positive selection (Additional file 1: Tables S3, S4). Among those, residue 307, located in the $\mathrm{C}$-terminal region, close to the seventh transmembrane domain, seems to diverge among diurnal and nocturnal snakes. In all diurnal species, this residue is occupied by an Arginine (R307), while a Lysine (K307) is found in most nocturnal snakes, including nocturnal colubrids from the Dipsadinae subfamily, and the viperids Bothrops jararaca and Protobothrops mucrosquamatus (Additional file 2). The viperid Crotalus durissus had a Threonine (T307), and primarily nocturnal colubrids from the subfamily Colubrinae (Lampropeltis getula, Arizona elegans and Cemophora coccinea) had R307, as found in diurnal species. Among the elapids, all considered diurnal species, only the fossorial coral snake Micrurus corallinus had K307, while the aquatic M. lemniscatus and the terrestrials Notechis scutatus and Pseudonaja textilis had R307. Both coral snakes, $M$. corallinus and $M$. lemniscatus, have diurnal activity, but diverge in the daily activity pattern observed in captivity, with a so-called unimodal pattern displayed by $M$. lemniscatus, with peak activity in the morning, and a bimodal pattern in $M$. corallinus, with peak activities in the morning and afternoon (Banci et al., manuscript in preparation). Those differences in activity pattern and habitat use may imply fine adjustments of the circadian entrainment, with variations in the expression levels and functions of the proteins associated with illuminance perception. Based on those observations, we suggest that residue 307 might be an interesting site for investigating specific mechanisms of melanopsin signaling and deactivation, in an attempt to search for divergences, which can ultimately drive differential activity patterns in snakes.

The melanopsin expression in all retinal nuclear layers of $T$. dorsatus, the outer and inner nuclear layers, and the ganglion cell layer, is curious and raises questions on 
specific functions and signaling pathways and projections of these photosensitive cells. We observed a large number of cones expressing melanopsin only in the retinal edge, close to the ora serrata, which suggests a regionalized co-expression of melanopsin and visual opsins. To our knowledge, this is the first study to report melanopsin expression in a cone population in non-teleost vertebrates. In teleost fishes, melanopsins are expressed in all retinal layers, including the photoreceptor layer [40, 41]. Davies et al. [40] suggested that the expression of the OPN4m-2 in cones might be related to light-dependent adaptations that extend the working range of these photoreceptors, by mediating the sustention of cytoplasmic calcium levels under bright light. Those studies described the expression of five Opn4 isoforms across all retinal neuron types of larvae and adult zebrafish [40, 41], indicating that a globally photosensitive retina would provide adaptation to a dynamic environmental light. In birds, both melanopsin paralogs Opn4m and Opn4x are expressed in different retinal cells with varied expression along the development [44, 45], which indicates distinct specific functions of the two melanopsins. In chicken retina, Opn4m is expressed only in a subpopulation of ganglion cells during all development stages, whereas Opn4x is expressed in ganglion cells in early development stages, and later it is rhythmically expressed in horizontal cells [34, 35, 44], indicating highly complex mechanisms for light detection, processing, and transmission to the brain [44]. In snakes, the melanopsin expression pattern, and distribution within the retina of diurnal and nocturnal species still has to be further investigated in order to understand the mechanisms underlying the control of daily activity pattern among the different species.

In conclusion, our analyses provide new insights into the evolution of the melanopsin genes in reptiles. Our results indicate that snakes have lost the Opn4m paralog and some species have lost extra-ocular photosensitive tissues, and we attribute this to a prolonged nocturnal $/ \mathrm{meso-}$ pic bottleneck in the early history of snake evolution. We also observed that the melanopsin gene is evolving under strong evolutionary constraint in snakes, and that daily activity pattern does not seem to have strong influence on the molecular evolution of melanopsin gene in snakes. Other features of the melanopsin function may be associated with the diversity of behavior regarding the daily activity patterns of the different species.

\section{Methods}

\section{Sample information}

Single specimens of 18 snake species from the Colubridae $(n=14)$, Elapidae $(n=2)$, and Viperidae $(n=2)$ families were provided by the Butantan Institute (Additional file 1: Table S1). Snakes were euthanized with a lethal injection of $30 \mathrm{mg} / \mathrm{kg}$ of sodium thiopental (Thionembutal). Individuals were kept under natural light conditions and were collected during the morning time. Animal procedures were in accordance with ethical principles of animal management and experimentation of the Brazilian Animal Experiment College (COBEA), and were approved by the Ethics Committee of Animal Research of the Butantan Institute, São Paulo, Brazil (777/10) and the Psychology Institute, University of São Paulo, Brazil $(9,635,070,717 ; 1,805,090,417)$.

\section{RNA extraction, polymerase chain reaction (PCR),} sequence alignment and gene tree reconstruction Eyes were enucleated and preserved in RNAlater (Life Technologies, Carlsbad, CA, USA), at $4{ }^{\circ} \mathrm{C}$. To investigate extra-retinal expression samples of iris, brain, liver, skeletal muscle and skin were collected from three colubrid species, Tomodon dorsatus, Sibynomorphus mikanii and Oxyrhopus guibei. Total RNA extraction and complementary DNA synthesis were performed as described previously [67].

Primers were designed using Primer 3 (v.0.4.0) [78], based on the predicted melanopsin sequences of the snakes Python bivittatus and Ophiophagus hanna (GenBank: XM_007429400.2 and AZIM01000714.1). Additionally, more specific primers were designed to amplify the melanopsin sequences of the species sampled in this study, based on the initial sequencing results (Additional file 1: Table S5). A pair of primers was also designed based on the Opn4m sequence of the red ear turtle Trachemys scripta elegans (GenBank: HM197714.1).

The procedures for polymerase chain reactions (PCRs), PCR products purification and DNA sequencing were performed as described previously [67]. Resulting sequences were visualized and aligned with BioEdit v7.0.9 [79] and Geneious v.9.1.3 (GeneMatters Corp.), using the iterative method of global pairwise alignment (MUSCLE and ClustalW) [80, 81]. The alignment included the 18 sequences generated in this study and 88 melanopsin sequences from other vertebrates obtained from GenBank (Additional file 1: Table S2). The melanopsin sequence of the lancelet (Branchiostoma belcheri) was used as outgroup (GenBank number: AB205400). Maximum Likelihood (ML) reconstruction was performed on the codon-match nucleotide alignment, using Garli v2.0 [82], and statistical support was estimated by non-parametric bootstrap [83], as described previously [67]. The model TVMef $+\mathrm{I}+\mathrm{G}$ was determined as the best-fit model of substitution for codon positions 1, 2, and 3, using Partition-Finder v.1.1.1 [84], and was used for ML reconstruction.

\section{Statistical analysis of molecular evolution}

To investigate the presence and type of selection acting on the melanopsin gene of diurnal and nocturnal snakes we applied a codon-based method, using the Codeml program 
from PAML v.4.7 [85]. We estimated the ratio of nonsynonymous $(\mathrm{dN})$ to synonymous $(\mathrm{dS})$ substitutions using random-sites models, branch models, branch-site models, and clade $(\mathrm{CmC})$ models [85-88]. The $\mathrm{dN} / \mathrm{dS}$ ratio $(\omega)$ indicates the type and magnitude of selection, where $\omega<1$ indicates purifying selection, $\omega \sim 1$ indicates neutral evolution, and $\omega>1$ indicates positive selection [85, 89]. Branch, branch-site and clade models allow appointing specific branches of interest as "foreground" and compare their $\omega$ rates with the $\omega$ estimated for the "background" branches [85]. Branch-site and $\mathrm{CmC}$ models were applied to test for divergence along specific branches of the tree [87]. Branch model allows only a single class of sites, and thus tests for average differences between clades. $\mathrm{CmC}$ allows two classes of sites across the tree to evolve conservatively $(0<\omega<1)$ and neutrally $(\omega=1)$, while a third site class is free to evolve differently among two or more partitions. The $\mathrm{CmC}$ null model, M2a rel, does not allow $\omega$ to diverge in the foreground clade [88]. Branch-site and random-sites models allow $\omega$ to vary among codon sites and to detect sites potentially under positive selection.

Likelihood ratio tests (LRTs) were used to compare competing models of evolution. The LRT statistic was computed as $2 \log$ likelihood difference between the two models and was tested against the $x^{2}$ distribution, where the degrees of freedom equal the difference between the numbers of parameters in the two nested models [85]. Bayesian information criterion (BIC) was also used to compare the models, and was computed as $-21+K \log$ $\mathrm{n}$, where $\mathrm{K}$ is the number of estimated parameters and $\mathrm{n}$ is the sample size. The lowest BIC score indicated the best model [90-92].

Branch model was applied to a broad data set including $O p n 4 x$ and $O p n 4 m$ melanopsin sequences from different vertebrate groups (Fig. 1; Additional file 1: Table S2; Sequences alignment: Additional file 3) to investigate the overall evolutionary patterns of Opn4x and Opn4m genes. The null branch model assumes the same $\omega$ ratio for all branches of the tree. We applied a two-partition model that assumes a $\omega_{0}$ for the Opn4m and a $\omega_{1}$ for the $O p n 4 x$, and a three-partition model, that assumes a $\omega_{0}$ for $O p n 4 m$, a $\omega_{1}$ for $O p n 4 x$, and a $\omega_{2}$ for the snake branch.

The random-site, branch-site and $\mathrm{CmC}$ models were applied to a smaller data set containing only the melanopsin sequences of snakes (Additional file 4). For those analyses, we used a snake phylogenetic tree congruent with that published previously (Fig. 2) [61]. Random-sites (M0, M1a, M2a, M2a_rel, M3, M7, M8a, and M8) [85, 88] were used to determine the overall selective patterns and to test for heterogeneous selection pressure among codon sites across all branches of the snake tree. LRT comparisons between random site models were used to test for variation in $\omega$ among sites (M3 vs. M0) and the presence and proportion of positively selected sites (M2a vs. M1a, M8 vs. M7, and M8 vs. M8a) [85, 93]. When LRTs were significant for positive selection, Bayes Empirical Bayes (BEB) was used to estimate posterior probabilities for site classes and identify amino acid sites under positive selection [85].

$\mathrm{CmC}$ models were designed to test two different hypothesis on the evolution of melanopsin of snakes, one based on the species phylogeny, and the other based on the species daily activity pattern (Fig. 2). For the phylogenetic hypothesis, we applied the following partition tests: a two-partition (Henophidian $\mathrm{x}$ Caenophidian snakes), a three-partition (Henophidian $\mathrm{x}$ Viperdiae $\mathrm{x}$ Colubridae+Elapidae) and a four-partition model (Henophidian $x$ Viperdiae $\mathrm{x}$ Colubridae $\mathrm{x}$ Elapidae). For daily activity pattern hypothesis, we tested a two-partition model, isolating all diurnal and all nocturnal species in separated clades, and compared to a three-partition model, which isolated the henophidian species Python bivitatus in a separate clade (Henophidia x Diurnal x Nocturnal), in order to test for divergences among the nocturnal henophidian and caenophidian lineages. Nested models were compared using LRT, and BIC was used to compare non-nested models, and determine whether diurnal or nocturnal habits display greater influence on melanopsin divergence than species relationships.

To analyze whether diurnal or nocturnal snake lineages might have experienced positive selection on any codon site, we used branch-site models [85] and implemented Model A (Model $=2$, NSsites $=2$ ), as an extension of the site-specific "neutral" model (M1) of Nielsen and Yang [94]. We also investigated for positive selected sites specifically in each snake family (Viperidae, Elapidae and Colubridae), by running two-partition branch-site models isolating each family as a foreground branch. The null models are the same as for Model A but with $\omega_{2}$ fixed at 1 for foreground branches. The proportions $\mathrm{p}_{0}$ and $\mathrm{p}_{1}$, as well as the ratio $\omega_{2}$, were estimated by maximum likelihood $[93,94]$.

\section{RNA in situ hybridization}

Whole eyes of two individuals of the diurnal T. dorsatus, were collected and fixed in 4\% paraformaldehyde (PFA) diluted in phosphate-buffered saline (PBS) (pH 7.2) treated with diethyl pyrocarbonate (DEPC) (Sigma-Aldrich, Darmstadt, Germany), for $12 \mathrm{~h}$, at $4^{\circ} \mathrm{C}$. Eyes were cryoprotected in $30 \%$ sucrose solution diluted in PBSDEPC for $72 \mathrm{~h}$, embedded in OCT (Tissue Tek Sakura Finetek Europe) and cryosectioned at $-25^{\circ} \mathrm{C}$. Radial sections at $12-\mu \mathrm{m}$ thicknesses were obtained in cryostat (CM1100 Leica, Nussloch, Germany) and collected onto Superfrost Plus microscope slides (VWR, UK).

Antisense and sense digoxigenin (DIG)-labelled RNA probes were synthesized by in vitro transcription using the SP6/T7 DIG RNA Labelling Kit (Roche, UK), 
following manufacturer's instruction. For antisense RNA probes, the T7 RNA polymerase promoter sequence 5' TAATACGACTCACTATAGGGAGA was added to the reverse primer, and for sense probes, to the forward primer. Sense probes were used as a control for nonspecific staining. cDNA of T. dorsatus was used as PCR templates for generating $750 \mathrm{bp}$ DIG-labeled RNA probes, synthesized with the primer pair OPN4_Oph_Fw2-5' TCCTGGTCTCCGTATGCT and OPN4_Oph_Rv2-5' GCCTTCCTTTGAGTAACAGGT. Amplicons were verified in a $1.2 \%$ agarose gel.

Retinal sections were post-fixed in PFA\%-DEPC for 5 min, permeabilized with proteinase $\mathrm{K}(0.1 \mu \mathrm{g} / \mathrm{ml})$ diluted in TE buffer ( $\mathrm{pH} 8)$-DEPC, for $15 \mathrm{~s}$ at room temperature (RT), washed in TE buffer for $15 \mathrm{~s}$ and prehybridized with ULTRAhyb ${ }^{\bullet}$ Ultrasensitive Hybridization Buffer (Ambion, UK) for $1 \mathrm{~h}$, at hybridization temperature. Slides were incubated with DIG-labelled riboprobes, diluted in preheated ULTRAhyb ${ }^{\bullet}$ Ultrasensitive Hybridization Buffer $(1 \mu \mathrm{g} / \mathrm{ml})$, covered with hybridization coverslip (Thermo Fischer Scientific) overnight, at $72^{\circ} \mathrm{C}$, in a humidified chamber. Coverslips were removed in pre-heated salinesodium citrate buffer $(0.2 \times \mathrm{SSC})$, and slides were washed three times in $0.2 \times$ SSC at $72^{\circ} \mathrm{C}$, and then twice in TBST buffer, at RT. Slides were blocked with $10 \%$ normal goat serum (NGS) (Sigma-Aldrich), diluted in TBST for $1 \mathrm{~h}$ and incubated overnight at $4^{\circ} \mathrm{C}$, in a humid chamber, with anti-digoxigenin antibody conjugated to alkaline phosphate (AP) (Roche, UK) (diluted 1:1000 in 1\% NGS in TBST buffer). Hybridized probes were detected using the BCIP/NBT (Roche). Slides were mounted in 50\% glycerol diluted in PBS and visualized in a Leica DM6B microscope (Leica Microsystems, Wetzlar, Germany). Images were obtained with a 40x (0.8 NA) objective, using the software LAS X Core (Leica Microsystems, Wetzlar, Germany).

\section{Additional files}

\section{Additional file 1: Tables S1-S5. (DOCX $31 \mathrm{~kb})$}

Additional file 2: Amino acid alignment of the melanopsin sequences of snakes and the lizard Podarcis sicula (GenBank: DQ013043.2). Horizontal bars show the location of the seven transmembrane domains predicted for the snake Python bivitattus (GenBank: XM_007429400.1). The gray boxes indicate the common features of melanopsins. (TIF $4171 \mathrm{~kb}$ )

Additional file 3: Nucleotide alignment of the Opn4x and Opn4m melanopsin sequences used for branch model analysis. (PDF $43 \mathrm{~kb}$ )

Additional file 4: Nucleotide alignment of melanopsin sequences of snakes used for random-site, branch-site and CmC models. (PDF 21 kb)

\section{Abbreviations}

BEB: Bayes Empirical Bayes; BIC: Bayesian information criterion; DEPC: Diethyl pyrocarbonate; DIG: Digoxigenin; dN: Nonsynonymous substitutions; dS: Synonymous substitutions; ipRGC: Intrinsically photosensitive retinal ganglion cells; ISH: In situ hybridization; LRT: Likelihood ratio test; ML: Maximum Likelihood; NGS: Normal goat serum; Opn4m: Mammalian-like melanopsin gene; Opn4x: Xenopus-like melanopsin gene; PBS: Phosphate- buffered saline; PCR: Polymerase chain reaction; PFA: Paraformaldehyde; RT: Room temperature; SSC: Sodium citrate buffer

\section{Acknowledgments}

We deeply appreciate Dr. Irene Yan and Dr. Ana Maria de Lauro Castrucci, for their kind support in experiment advice and discussions. We thank Butantan Institute and members of the Herpetology Laboratory for providing the animals. We also thank the two anonymous reviewers for advice and comments on previous versions of this manuscript.

\section{Authors' contributions}

Research conception and design: EH, DMOB, DFV; Data collection: EH, VK. Statistical analysis: EH; Analysis and interpretation: EH, DMOB, DFV; Drafting the paper: EH; Critical revision of the article: DMOB, DFV; Obtained funding: $\mathrm{EH}, \mathrm{DFV}$. All authors have read and approved the final manuscript.

\section{Funding}

This study was funded by the Foundation of Research Support in the State of São Paulo (FAPESP), with fellowships to EH (2010/51670-8; 2014/25743-9), and grants to DFV (2008/58731-2; 2014/26818-2; 2009/06026-6). DFV is a CNPq 1 A Productivity Fellow.

The funding bodies played no role in study design, data collection, analysis, interpretation of data, and in writing the manuscript.

\section{Availability of data and materials}

Sequences generated in this study were deposit at the GenBank ${ }^{\circledast} \mathrm{NIH}$ genetic sequence database (accession numbers MN241125- MN241142).

\section{Ethics approval}

Animal procedures were in accordance with ethical principles of animal management and experimentation established by the Brazilian Animal Experiment College (COBEA) and were approved by the Ethics Committee of Animal Research of the Butantan Institute (777/10), and the Psychology Institute, University of São Paulo (9635070717; 1805090417).

\section{Consent for publication}

Not applicable.

\section{Competing interests}

The authors declare that they have no competing interests.

\section{Author details}

'Departamento de Psicologia Experimental, Instituto de Psicologia, Universidade de São Paulo, Av. Professor Mello Moraes, 1721, Bloco A - Sala D9. Butantã, São Paulo, SP 05508-030, Brazil. ${ }^{2}$ Instituto Israelita de Ensino e Pesquisa Albert Einstein, São Paulo, Brazil. ${ }^{3}$ Christian Brothers University, Memphis, TN, USA.

Received: 18 February 2019 Accepted: 22 August 2019

Published online: 28 August 2019

\section{References}

1. Shand J, Foster RG. The extraretinal photoreceptors of non-mammalian vertebrates. In: Archer SN, Djamgoz MBA, Loew ER, Partridge JC, Vallerga S, editors. Adaptive mechanisms in the ecology of vision. Dordrecht: Kluwer Academic Publisher; 1999. p. 197-222

2. Blackshaw S, Snyder SH. Parapinopsin, a novel catfish opsin localized to the parapineal organ, defines a new gene family. J Neurosci. 1997;17:8083-92.

3. Soni BG, Foster RG. A novel and ancient vertebrate opsin. FEBS Lett. 1997; 406:279-83.

4. Kojima D, Mano H, Fukada Y. Vertebrate ancient-long opsin: a greensensitive photoreceptive molecule present in zebrafish deep brain and retinal horizontal cells. J Neurosci. 2000;20:2845-51.

5. Provencio I, Jiang G, De Grip WJ, Hayes WP, Rollag MD. Melanopsin: an opsin in melanophores, brain, and eye. Proc Natl Acad Sci U S A. 1998a:95:340-5.

6. Provencio I, Rodriguez IR, Jiang GS, Hayes WP, Moreira EF, Rollag MD. A novel human opsin in the inner retina. J Neurosci. 2000;20:600-5.

7. Hankins MW, Davies WIL, Foster RG. The Evolution of Non-visual Photopigments in the Central Nervous System of Vertebrates. In: Hunt DM, Hankins MW, Collin SP, Marshall NJ, editors. Evolution of Visual and Non- 
visual Pigments. New York: Springer New York Heidelberg Dordrecht London; 2014. p. 65-103.

8. Burns ME, Baylor DA. Activation, deactivation, and adaptation in vertebrate photoreceptor cells. Ann Rev Neurosci. 2001;24:779-805.

9. Menon ST, Han M, Sakmar TP. Rhodopsin: structural basis of molecular physiology. Physiol Rev. 2001;81(4):1659-88.

10. Walker MT, Brown RL, Cronin TW, Robinson PR. Photochemistry of retinal chromophore in mouse melanopsin. Proc Nat Acad Sci. 2008;105(26):8861-5.

11. Burns ME, Lamb TD. Visual transduction by rod and cone photoreceptors. In: Chalupa LM, Werner JS, editors. The visual neurosciences. Cambridge: MIT Press; 2003. p. 215-233.

12. Hardie RC. Phototransduction: shedding light on translocation. Curr Biol. 2013:13:R775-7.

13. Foster RG, Provencio I, Hudson D, Fiske S, DeGrip W, Menaker M. Circadian photoreception in the retinally degenerate mouse (rd/rd). J Comp Physiol A. 1991;169(1):39-50.

14. Panda S, Sato TK, Castrucci AM, Rollag MD, Degrip WJ, Hogenesch JB, Provencio I, Kay SA. Melanopsin (Opn4) requirement for normal lightinduced circadian phase shifting. Science. 2002;298:2213-6.

15. Ruby NF, Brennan TJ, Xie X, Cao V, Franken P, Heller HC, O'Hara BF. Role of melanopsin in circadian responses to light. Science. 2002;298:2211-3.

16. Hattar S, Lucas RJ, Mrosovsky N, Thompson S, Douglas RH, Hankins MW, Lem J, Biel M, Hofmann F, Foster RG, Yau KW. Melanopsin and rod-cone photoreceptive systems account for all major accessory visual functions in mice. Nature. 2003;424:76-81.

17. Lucas RJ, Douglas RH, Foster RG. Characterization of an ocular photopigment capable of driving pupillary constriction in mice. Nat Neurosci. 2001:4:621-6.

18. Lucas RJ, Hattar S, Takao M, Berson DM, Foster RG, Yau KW. Diminished pupillary light reflex at high irradiances in melanopsin-knockout mice. Science. 2003;299:245-7.

19. Lucas RJ, Freedman MS, Munoz M, Garcia-Fernandez JM, Foster RG. Regulation of the mammalian pineal by non-rod, non-cone, ocular photoreceptors. Science. 2011;284:505-7.

20. Berson DM, Dunn FA, Takao M. Phototransduction by retinal ganglion cells that set the circadian clock. Science. 2002:295(5557):1070-3.

21. Hattar S, Liao HW, Takao M, Berson DM, Yau KW. Melanopsin-containing retinal ganglion cells: architecture, projections, and intrinsic photosensitivity. Science. 2002;295:1065-70.

22. Rollag MD, Berson DM, Provencio I. Melanopsin, ganglion-cell photoreceptors, and mammalian photoentrainment. J Biol Rhythm. 2003;18:227-34.

23. Hankins MW, Peirson SN, Foster RG. Melanopsin: an exciting photopigment. Trends Neurosci. 2008;31:27-36.

24. Bailes HJ, Lucas RJ. Melanopsin and inner retinal photoreception. Cell Mol Life Sci. 2010:67:99-111.

25. Muller LP, Do MT, Yau KW, He S, Baldridge WH. Tracer coupling of intrinsically photosensitive retinal ganglion cells to amacrine cells in the mouse retina. J Comp Neurol. 2010;518:4813-24.

26. Gamlin PD, McDougal DH, Pokorny J, Smith VC, Yau KW, Dacey DM. Human and macaque pupil responses driven by melanopsin-containing retinal ganglion cells. Vis Res. 2007;47:946-54.

27. Hatori M, Panda S. The emerging roles of melanopsin in behavioral adaptation to light. Trends Mol Med. 2010;16:435-46.

28. Bellingham J, Chaurasia SS, Melyan Z, Liu C, Cameron MA, Tarttelin EE, luvone PM, Hankins MW, Tosini G, Lucas RJ. Evolution of melanopsin photoreceptors: discovery and characterization of a new melanopsin in nonmammalian vertebrates. PLoS Biol. 2006;4:e254.

29. Borges R, Johnson WE, O'Brien SJ, Vasconcelos V, Antunes A. The role of gene duplication and unconstrained selective pressures in the melanopsin gene family evolution and vertebrate circadian rhythm regulation. PLoS One. 2012;7:e52413.

30. Peirson S, Bovee-Geurts P, Lupi D, Jeffery G, DeGrip W, Foster RG. Expression of the candidate circadian photopigment melanopsin (Opn4) in the mouse retinal pigment epithelium. Mol Brain Res. 2004;123:132-5.

31. Bellingham J, Whitmore D, Philp AR, Wells DJ, Foster RG. Zebrafish melanopsin: isolation, tissue localisation and phylogenetic position. Mol Brain Res. 2002:107:128-36.

32. Jenkins A, Munoz M, Tarttelin EE, Bellingham J, Foster RG, Hankins MW. VA opsin, melanopsin, and an inherent light response within retinal interneurons. Curr Biol. 2003;13:1269-78.

33. Drivenes O, Soviknes AM, Ebbesson LO, Fjose A, Seo HC, Helvik JV. Isolation and characterization of two teleost melanopsin genes and their differential expression within the inner retina and brain. J Comp Neurol. 2003:456:84-93.

34. Bailey MJ, Cassone VM. Melanopsin expression in the chick retina and pineal gland. Brain Res Mol Brain Res. 2005;134:345-8.

35. Chaurasia SS, Rollag MD, Jiang G, Hayes WP, Haque R, Natesan A, Zatz M, Tosini G, Liu C, Korf HW, luvone PM, Provencio I. Molecular cloning, localization and circadian expression of chicken melanopsin (Opn4): differential regulation of expression in pineal and retinal cell types. J Neurochem. 2005:92:158-70.

36. Grone BP, Sheng Z, Chen CC, Fernald RD. Localization and diurnal expression of melanopsin, vertebrate ancient opsin, and pituitary adenylate cyclase-activating peptide mRNA in a teleost retina. J Biol Rhythm. 2007:22:558-61.

37. Tomonari S, Takagi A, Noji S, Ohuchi H. Expression pattern of the melanopsin-like (cOpn4m) and VA opsin-like genes in the developing chicken retina and neural tissues. Gene Expr Patterns. 2007;7:746-53.

38. Cheng N, Tsunenari T, Yau KW. Intrinsic light response of retinal horizontal cells of teleosts. Nature. 2009;460:899-903.

39. Walls GL. The vertebrate eye and its adaptive radiation. Bloomfield Hills: Cranbook Inst of Science; 1942

40. Davies WI, Zheng L, Hughes S, Tamai TK, Turton M, Halford S, Foster RG, Whitmore D, Hankins MW. Functional diversity of melanopsins and their global expression in the teleost retina. Cell Mol Life Sci. 2011;68:4115-32.

41. Matos-Cruz V, Blasic J, Nickle B, Robinson PR, Hattar S, Halpern ME. Unexpected diversity and photoperiod dependence of the zebrafish melanopsin system. PLoS One. 2011;6(9):e25111.

42. Gooley JJ, Lu J, Chou TC, Scammell TE, Saper CB. Melanopsin in cells of origin of the retinohypothalamic tract. Nat Neurosci. 2001;4:1165.

43. Thapan K, Arendt J, Skene DJ. An action spectrumfor melatonin suppression: evidence for a novel non-rod, non-conephotoreceptor system in humans. J Physiol. 2001;535:261-7.

44. Verra DM, Contín MA, Hicks D, Guido ME. Early onset and differential temporospatial expression of melanopsin isoforms in the developing chicken retina. IOVS. 2011:52(8):5111-20.

45. Lima LH, M. SA, Isoldi MC, A. VM, Castrucci AM. Melanopsin in chicken melanocytes and retina. Biol Rhythm Res. 2006;37:393-404.

46. Dearworth JR Jr, Selvarajah BP, Kalman RA, Lanzone AJ, Goch AM, Boyd AB, Goldberg LA, Cooper LJ. A mammalian melanopsin in the retina of a fresh water turtle, the red-eared slider (Trachemys scripta elegans). Vis Res. 2011; 51(2):288-95.

47. Frigato E, Vallone D, Bertolucci C, Foulkes NS. Isolation and characterization of melanopsin and pinopsin expression within photoreceptive sites of reptiles. Naturwissenschaften. 2006:93:379-85.

48. Crowe-Riddell JM, Simões BF, Partridge JC, Hunt DM, Delean S, Schwerdt JG, Breen J, Ludington A, Gower D, Sanders KL. Phototactic tails: evolution and molecular basis of a novel sensory trait in sea snakes. Mol Ecol. 2019;28: 2013-28.

49. Castoe TA, et al. The Burmese python genome reveals the molecular basis for extreme adaptation in snakes. Proc Natl Acad Sci U S A. 2013;110(51): 20645-50.

50. Emerling CA. Genomic regression of claw keratin, taste receptor and lightassociated genes provides insights into biology and evolutionary origins of snakes. Mol Phyl Evol. 2017:115:40-9.

51. Perry BW, et al. Molecular adaptations for sensing and securing prey and insight into amniote genome diversity from the garter snake genome. Gen Biol Evol. 2018;10(8):2110-29.

52. Schott RK, Van Nynatten A, Card DC, Castoe TA, Chang BS. Shifts in selective pressures on snake phototransduction genes associated with photoreceptor transmutation and dim-light ancestry. Mol Biol Evol. 2018;35(6):1376-89.

53. Uetz P. (editor). The Reptile Database, http://www.reptile-database.org, Accessed 24 July 2019

54. Lillywhite HB. Temperature, energetics and physiological ecology. In: Seigel RA, Collins JT, Novak SS, editors. Snakes: ecology and evolutionary biology. New York: McGraw-Hill; 1987. p. 422-77.

55. Gibbons JW, Semlitsch RD. Activity patterns. In: Seigel RA, Collins JT, Novak SS, editors. Snakes: ecology and evolutionary biology. New York: McGrawHill; 1987. p. 184-209.

56. Marques OAV, Eterovic A, Sazima I. Serpentes da Mata Atlântica: guia ilustrado para a Serra do Mar. Holos. 2001;v.1:184.

57. Torello-Viera NF, Marques OAV. Daily activity of Neotropical Dipsadids snakes. S Am J Herpetol. 2017;12(2):128-35. 
58. Provencio I, Cooper HM, Foster RG. Retinal projections in mice with inherited retinal degeneration: implications for circadian photoentrainment. J Comp Neurol. 1998;395:417-39.

59. Terakita A, Yamashita T, Shichida Y. Highly conserved glutamic acid in the extracellular IV-V loop in rhodopsins acts as the counterion in retinochrome, a member of the rhodopsin family. Proc Natl Acad Sci U S A. 2000;97:14263-7.

60. Bockaert J, Pin JP. Molecular tinkering of G protein-coupled receptors: an evolutionary success. EMBO J. 1999;18:1723-9.

61. Pyron RA, Burbrink FT, Wiens JJ. A phylogeny and revised classification of Squamata, including 4161 species of lizards and snakes. BMC Evol Biol. 2013;13:93.

62. Simões BF, Sampaio FL, Douglas RH, Kodandaramaiah U, Casewell NR, Harrison RA, Hart NS, Partridge JC, Hunt DM, Gower DJ. Visual pigments, ocular filters and the evolution of snake vision. Mol Biol Evol. 2016:33(10):2483-95

63. Davies WL, Cowing JA, Bowmaker JK, Carvalho LS, Gower DJ, Hunt DM. Shedding light on serpent sight: the visual pigments of henophidian snakes. J Neurosci. 2009;29:7519-25.

64. Simões BF, Sampaio FL, Jared C, Antoniazzi MM, Loew ER, Bowmaker JK Rodriguez A, Hart NS, Hunt DM, Partridge JC, Gower DJ. Visual system evolution and the nature of the ancestral snake. J Evol Biol. 2015:28:1309-20.

65. Schott RK, Müller J, Yang CGY, Bhattacharyya N, Chan N, Xu M, Morrow JM, Ghenu AH, Loew ER, Tropepe $V$, Chang BSW. Evolutionary transformation of rod photoreceptors in the all-cone retina of a diurnal garter snake. Proc Natl Acad Sci U S A. 2016;113:356-61.

66. Bhattacharyya N, Darren B, Schott RK, Tropepe V, Chang BS. Cone-like rhodopsin expressed in the all cone retina of the colubrid pine snake as a potential adaptation to diurnality. J Exp Biol. 2017;220(Pt 13):2418-25.

67. Hauzman E, Bonci DMO, Suárez-Villota EY, Neitz M, Ventura DF. Daily activity patterns influence retinal morphology, signatures of selection, and spectral tuning of opsin genes in colubrid snakes. BMC Evol Biol. 2017;17(1):249.

68. Emerling CA. Archelosaurian color vision, parietal eye loss, and the crocodylian nocturnal bottleneck. Mol Biol Evol. 2017;34(3):666-76.

69. Pires SS, Shand J, Bellingham J, Arrese C, Turton M, Peirson S, Foster RG, Halford S. Isolation and characterization of melanopsin (Opn4) from the Australian marsupial Sminthopsis crassicaudata (fat-tailed dunnart). Proc R Soc B. 2007;274:2791-9.

70. Ronan M, Bodznick D. Behavioral and neurophysiological demonstration of a lateralis skin photosensitivity in larval sea lampreys. J Exp Biol. 1991;161:97-117.

71. Patzner RA. Experimental studies on the light sense in the hagfish, Eptatretus burgeri and Paramyxine atami (Cyclostomata). Helgoländer Meeresun. 1978;31(1-2):180-90.

72. Sayle MH. The reactions of Necturus to stimuli received through the skin. J An Behav. 1916;6(2):81-102.

73. Underwood G. A contribution to the classification of snakes. London: Trustees of the British Museum (Natural History); 1967.

74. Wong R. Morphology and distribution of neurons in the retina of the American garter snake (Thamnophis sirtalis). J Comp Neurol. 1989;283:597-601.

75. Sillman AJ, Govardowskii VI, Rohlich P, Southard JA, Loew ER. The photoreceptors and visual pigments of the garter snake (Thamnophis sirtalis): a microspectrophotometric, scanning electron microscopic and immunocytochemical study. J Comp Physiol A. 1997;181:89-101.

76. Hart NS, Coimbra JP, Collin SP, Westhoff G. Photoreceptor types, visual pigments, and topographic specializations in the retinas of hydrophiid sea snakes. J Comp Neurol. 2012;520:1246-61.

77. Hauzman E, Bonci DMO, Grotzner SR, Mela M, Liber AMP, Martins SL, Ventura DF. Comparative study of photoreceptor and retinal ganglion cell topography and spatial resolving power in Dipsadidae snakes. Brain Behav Evol. 2014;84:197-213.

78. Untergrasser A, Cutcutache I, Koressaar T, Ye J, Faircloth BC, Remm M, Rozen SG. Primer3 - new capabilities and interfaces. Nucleic Acids Res 2012:40:e115

79. Hall TA. BioEdit: a user-friendly biological sequence alignment editor and analysis program for windows 95/98/NT. In nucleic acids symposium series. London. 1999:41:95-8.

80. Thompson JD, Higgins DG, Gibson TJ. Clustal W: improving the sensitivity of progressive multiple sequence alignment through sequence weighting, position specific gap penalties and weight matrix choice. Nucleic Acid Res. 1994;22:4673-80.
81. Edgar RC. MUSCLE: multiple sequence alignment with high accuracy and high throughput. Nucleic Acids Res. 2004;32:1792-7.

82. Bazinet AL, Zwickl DJ, Cummings MP. A gateway for phylogenetic analysis powered by grid computing featuring GARLI 2.0. Syst Biol. 2014:63:812-8.

83. Felsenstein J. Confidence limits on phylogenies: an approach using the bootstrap. Evolution. 1985;39:783-91.

84. Lanfear R, Calcott B, Ho SYW, Guindon S. PartitionFinder: combined selection of partitioning schemes and substitution models for phylogenetic analyses. Mol Biol Evol. 2012;29:1695-701.

85. Yang Z. PAML 4: phylogenetic analysis by maximum likelihood. Mol Biol Evol. 2007;24:1586-91.

86. Bielawski JP, Yang Z. A maximum likelihood method for detecting functional divergence at individual codon sites, with application to gene family evolution. J Mol Evol. 2004;59(1):121-32.

87. Zhang J, Nielsen R, Yang Z. Evaluation of an improved branch-site likelihood method for detecting positive selection at the molecular level. Mol Biol Evol. 2005;22(12):2472-9.

88. Weadick CJ, Chang BSW. An improved likelihood ratio test for detecting site-specific functional divergence among clades of protein-coding genes. Mol Biol Evol. 2012;29(5):1297-300.

89. Yang Z. Likelihood ratio tests for detecting positive selection and application to primate lysozyme evolution. Mol Biol Evol. 1998;15:568-73.

90. Schwarz G. Estimating the dimension of a model. Ann Stat. 1978;6:461-4.

91. Posada D, Buckley TR. Model selection and model averaging in phylogenetics: advantages of akaike information criterion and Bayesian approaches over likelihood ratio tests. Syst Biol. 2004:53:793-808.

92. Seo TK, Kishino H. Statistical comparison of nucleotide, amino acid, and codon substitution models for evolutionary analysis of protein-coding sequences. Syst Biol. 2009;58:199-210.

93. Yang Z, Nielsen R, Goldman N, Pedersen AM. Codon-substitution models for heterogeneous selection pressure at amino acid sites. Genetics. 2000;155:431-49.

94. Nielsen R, Yang Z. Likelihood models for detecting positively selected amino acid sites and applications to the HIV-1 envelope gene. Genetics. 1998;148:929-36.

\section{Publisher's Note}

Springer Nature remains neutral with regard to jurisdictional claims in published maps and institutional affiliations.

\section{Ready to submit your research? Choose BMC and benefit from:}

- fast, convenient online submission

- thorough peer review by experienced researchers in your field

- rapid publication on acceptance

- support for research data, including large and complex data types

- gold Open Access which fosters wider collaboration and increased citations

- maximum visibility for your research: over $100 \mathrm{M}$ website views per year

At $\mathrm{BMC}$, research is always in progress.

Learn more biomedcentral.com/submissions 